\title{
Healthcare-associated hand injuries
}

\author{
Keywords: hand, bloodborne viruses, needlestick, healthcare, \\ surgery
}

Abbreviations: WHO, World health organisation; FDP, flexor digitorum profundus; FDS, flexor digitorum superficialis

\section{Introduction}

Healthcare workers are exposed to blood and other body fluids in the course of their work and are therefore at risk of bloodborne viruses. The World Health Organisation (WHO) estimates 3 million receive percutaneous exposures to blood borne viruses each year, $90 \%$ being in the developing world. ${ }^{1}$ Whilst needlestick injuries, despite the majority being easily preventable, are well described injuries within healthcare settings, a significant number of medical devices and instruments also pose a risk to users, especially if the correct training has not been undertaken for their safe use. The number of non-needlestick sharps injuries is unknown, as these are less likely to be recorded. ${ }^{2}$ Workers in operating theatres are at increased risk of injury due to the more frequent usage of such instruments, ${ }^{3}$ and it is estimated these account for $7-8 \%$ of all sharps injuries. ${ }^{4}$ Between 2004-2013, of all cases reported to Public Health England involving a sharp instrument, 427/3163 (14\%) involved non-needle injuries, ${ }^{5}$ and these are commonly underreported. ${ }^{3}$

Penetrating injuries to the hand, which not only carry the risk of transmission of blood born viruses, present a risk of deeper structural damage which can cause significant injury. Damage to tendons and nerves within the hand, despite early diagnosis and surgical intervention, can result in poor functional outcomes. Tendon injuries require a lengthy period of physiotherapy to prevent stiffness and maintain full range of movement whilst not risking rupture of the newly repaired tendon from excessive load on the digit. Digital nerve recovery is unpredictable and can depend on patient age or comorbidities resulting in sensory deficit if incomplete recovery. ${ }^{6}$ Visible blood increases risk of transmission and these instruments are likely to be much more visible heavily contaminated than needles. This case series highlights the potential severity of healthcare-associated and injuries and factors to consider preventing further injury.

\section{Case series}

\section{Case I}

A 62-year-old right-handed midwife sustained a penetrating injury to her left index finger whilst attempting to remove a scalpel blade from its handle. This caused a laceration to the volar aspect of the digit, and more significantly a zone 2 division of flexor digitorum profundus (FDP) and the radial slip of flexor digitorum superficialis (FDS) tendons. These were repaired surgically and a period of hand therapy was commenced. The patient has not yet returned to work and even after 8 weeks of hand therapy still have ongoing stiffness and limited extension of both the little and ring fingers. On examination there is thickening of scar tissue extending radially across the palm which may represent post-traumatic dupuytrens disease. Flexor tenolysis to the little finger was performed, revealing adhesion to the A3 pulley, under which the repair was situated. Following the release,
Volume 8 Issue 4 - 2020

Tom Challoner, Rajive Jose

Department of Plastic Surgery, Queen Elizabeth Hospital, United Kingdom

Correspondence: Tom Challoner, Department of Plastic Surgery, Queen Elizabeth Hospital, Birmingham, United Kingdom, BI5 2TH, Email Tomchalloner@gmail.com

Received: May 16, 2020 | Published: October 19, 2020

the extension of the ring finger improved and the patients returned to work. Further aggressive hand therapy is required to maintain the range of movement gained from the tenolysis.

\section{Case 2}

A 29-year-old right-handed theatre nurse was trying to mount a weck knife, used for excising layers of skin most commonly in burns theatres. Although a correct procedure exists for mounting the blade, this was not followed due to ease and speed of an alternative technique. The blade slipped and lacerated her left index finger sustaining a zone 2 injury to both flexor tendons and the ulnar digital nerve. These were successfully repaired surgically and commenced early active mobilisation to prevent scar tissue formation. However due to reaching plateau in tendon rehabilitation, the patient underwent a tenolysis procedure and neurolysis, a release of the digital nerve from scar tissue, followed by wrapping in an expendable vein from the volar wrist to limit irritation and further scar tissue formation. This secondary procedure was successful, and although there is a limitation by $5^{0}$ at the proximal interphalangeal joint, and the sensation $7 / 10$ along the affected side, the patient has returned to work with full function of the hand.

\section{Case 3}

A 44-year-old right-handed General Practitioner, after performing a routine punch biopsy Figure 1, left the instrument on the trolley whilst gathering the sharps. She was distracted by the patient re-entering the room, and whilst she would normally have an assistant to help dispose of the sharps, on this day she did not. When she returned to the trolley, she attempted to tidy up and was scrunching up some paper, not realising the punch was inside, and forcibly penetrated her left middle finger. This left a circular skin defect over the middle phalanx and a multi-level zone 1 injury to FDP. She underwent an operation to repair the flexor tendon, and remains on amended duties at work due to limitations in movement after 3 months of rehabilitation.

\section{Discussion}

Following a sharps injury, as with a needlestick injury, initial first aid should include thoroughly cleaning the wound with running water, and the wound should be encouraged to bleed. ${ }^{7}$ It can be dressed prior 
to formal assessment. An incident report should be filled, which should initiate discussion of how a repeat incident can be prevented. Risk assessment is undertaken with the local occupational health department to ascertain the likely transmission of blood borne viruses, and blood samples are taken from the patient and staff member injured for the same. These procedures were carried out in all of the described cases.

A study by Tokars et al., ${ }^{8}$ identified surgeons being most at risk during surgical procedures, and that members of the scrub team had relatively low risk. Recent advances in techniques and awareness of risk may have reduced the intraoperative risk. Our cases highlight that risk is not simply from passing instruments, as our cases were from preparing instruments or distraction following a procedure. Studies within the United Kingdom, suggest $41 \%$ of doctors have suffered a sharp injury, $44 \%$ of which are surgeons, compared to $45 \%$ of nursing staff, 3,5 however underreporting is common place, due to either low perceived risk or fear of blame. ${ }^{2}$ Nursing staff remain at most risk outside of surgical environments due to their increased exposure and usage of sharps.

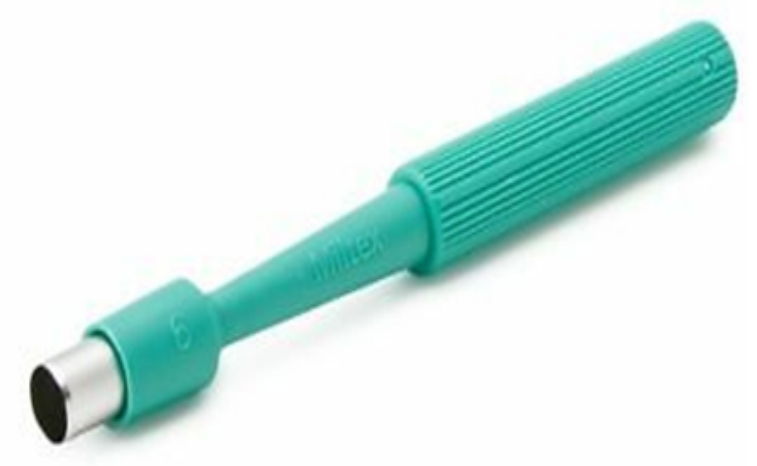

Figure I Punch biopsy tool, demonstrating circular metal cutting edge.

The cases discussed present several factors which highlight the significance of injuries sustained from medical instruments. They are often designed to penetrate skin easily and therefore are extremely sharp. Poorly maintained equipment also may lead to more resistance on assembly, and increase the risk of injury. Problematic equipment should be highlighted during the World Health Organisation mandatory checklist, however often this is overlooked and perhaps stiff or poorly fitting equipment is accepted as normal. Therefore, these tools remain in circulation for future usage.

Whilst one cannot completely prevent sharps related injuries in healthcare practice, there are steps one can take to minimise the incidence of these events. In the first case, use of a blade remover Figure 2 would have prevented the injury which happened while trying to remove the blade from the scalpel. Although products exist for the safe disposal of sharps, anecdotally scrub nurses prefer to rely on their own trusted methods, feeling that the devices are potentially more hazardous. In the second case, the correct technique of mounting the Weck blade was not followed. These blades are extremely sharp and attempting to push the blade using fingers is risky. The correct method avoiding manual contact with the blade should be followed. The third case illustrates the importance of disposing off sharps in the sharps bin immediately after use. Leaving them in a general tray after use is unsafe from injury risk as well as a contamination risk.

Training is vitally important when using the instruments discussed, as although assembly of blades may seem simple, there are often tried and tested safe methods of assembly, which can prevent injury. It is the responsibility of the employer and supervising staff to ensure the correct procedures are followed. ${ }^{9}$ Several studies have attempted to analyse the financial implications of these injuries, from either direct, such as laboratory testing and post-exposure treatment, or indirect, being the cost of appointments, staff absence and compensation. A systematic review by Mannocci et al., ${ }^{10}$ estimated these costs average between $\$ 650-750$, without costs of ongoing medication or review. Cases such as those described with injuries requiring surgical intervention would cost significantly more, including theatre visits, and multiple outpatients' appointments, as well as significant periods of staff absence to cover. The safe disposal of sharps must also be of paramount importance following the completion of a procedure. It should be of primary importance to dispose of sharps as soon as possible, as any delay, coupled with distraction such as in the case highlighted can lead to injury. This can be compounded by staff shortages and straying from the routine, taking on additional responsibilities and being unfamiliar with locations and protocols. Whilst most needlestick injuries do not prevent return to work, sharps injuries in this context can prolong recovery to a point where hand function is at a level it will not compromise safety.

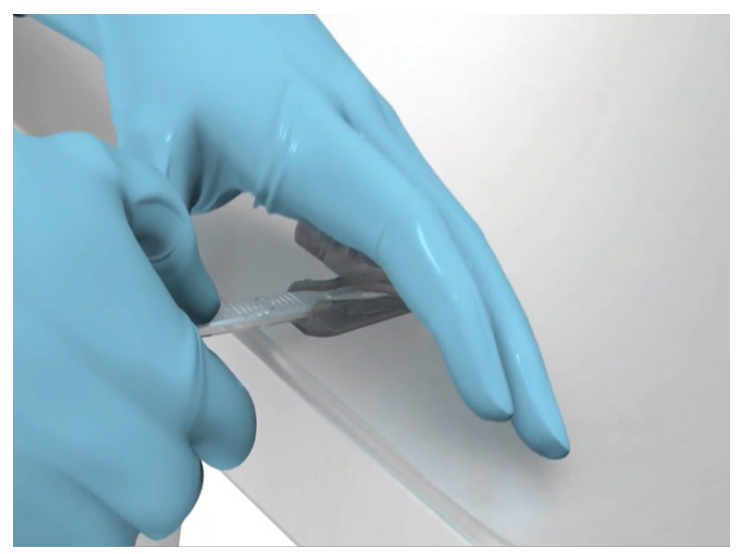

Figure 2 The use of a disposable blade remover. This avoids potential contact with the blade. Photo taken from Swann-Morton catalogue, Sheffield, UK.

These cases require both the initial surgical repair but also several months of hand therapy and splinting with the aim to protect the repair and restore function. This can mean a prolonged absence from work due to initially health and safety with an unhealed wound and need for controlled mobility of the affected digit. There is a limitation in the ability to drive to the place of work, lack of availability of amended duties in the workplace, as most healthcare staff rely on hand function to examine or treat patients, or reduced ability to maintain hygiene and regular hand washing with the splint in place. The average time to return to work is about 10 weeks following flexor tendon injury but as full mobility, tendon strength and the safety of heavy lifting is limited until 12-16 weeks this can be longer. A full occupational health assessment is required on return to work to ensure safety on returning to routine work.

\section{Conclusion}

Whilst needlestick injuries are well described and a well described pathway exists within all healthcare organisations, significant care must be taken within environments performing surgical procedures to the correct usage of instruments. This subset of injuries is underrepresented in medical literature and serves to highlight other risks to sharps other than infectious consequences. This discussion 
emphasises the importance of incidence reporting as this can serve to highlight shortcomings in equipment or processes.

\section{Acknowledgments}

None.

\section{Conflicts of interest}

The authors declare that there have no conflicts of interest.

\section{Funding}

None.

\section{References}

1. World Health Organization. Aide-memoire for a strategy to protect health workers from infection with bloodborne viruses. World health organization. 2013.

2. EPSU-HOSPEEM. Promotion and support of implementation of directive 2010/32/EU on the prevention of sharps injuries in the hospital and health care sector. 2013.

3. Thomas WJC, Murray JRD. The incidence and reporting rates of needlestick injury amongst UK surgeons. Ann R Coll Surg Eng. 2009;91(1):1217.
4. Fuentes H, Collier J, Sinnott M, et al. "Scalpel safety": modelling the effectiveness of different safety devices' ability to reduce scalpel blade injuries. Int J Risk Saf Med. 2008;20:83-89.

5. Eye of the needle. United Kingdom surveillance of significant occupational exposures to bloodborne viruses in healthcare workers. Public Health England, London. 2020.

6. Kim JS, Bonsu NY, Leland HA, et al. A systematic review of prognostic factors for sensory recovery after digital nerve reconstruction. Ann Plast Surg. 2018;80(5S Suppl 5):S311-S316.

7. NHS UK. What should I do if I injure myself with a needle? 2018.

8. Tokars JI, Bell DM, Culver DH, et al. Percutaneous injuries during surgical procedures. JAMA. 1992;267(21):2899-2904.

9. Health and safety executive. Health and safety (sharp instruments in healthcare) regulations 2013. guidance for employers and employees.

10. Mannocci A, De Carli G, Di Bari V, et al. How much do needlestick injuries cost? a systematic review of the economic evaluations of needlestick and sharps injuries among healthcare personnel. Infect Control Hosp Epidemiol. 2016;37(6):635-646. 